Rabaska

Revue d'ethnologie de l'Amérique française

Mooney, Fraser. Jerome. Solving the Mystery of Nova Scotia's

Silent Castaway. Halifax, Nimbus, 2008, 178 p. ISBN

978-1-55109-686-5

\title{
Caroline-Isabelle Caron
}

Volume 7, 2009

URI : https://id.erudit.org/iderudit/038371ar

DOI : https://doi.org/10.7202/038371ar

Aller au sommaire du numéro

Éditeur(s)

Société québécoise d'ethnologie

ISSN

1703-7433 (imprimé)

1916-7350 (numérique)

Découvrir la revue

Citer ce compte rendu

Caron, C.-I. (2009). Compte rendu de [Mooney, Fraser. Jerome. Solving the Mystery of Nova Scotia's Silent Castaway. Halifax, Nimbus, 2008, 178 p. ISBN 978-1-55109-686-5]. Rabaska, 7, 221-223. https://doi.org/10.7202/038371ar d'utilisation que vous pouvez consulter en ligne.

https://apropos.erudit.org/fr/usagers/politique-dutilisation/ 
populaires, Porter, dir., 1984). L'important, ce n'est pas tellement d'avoir la réponse à ces questions mais de se les poser. Surtout lorsqu'on prétend produire sur le sujet un ouvrage de référence. Sans porter de jugement de valeur sur la qualité intrinsèque des œuvres présentées dans l'ouvrage d'Adrien Levasseur, il faut bien reconnaître qu'à côté de la production de maîtres reconnus, souvent associée à une longue tradition familiale, plusieurs œuvres détonnent parce que, justement, sans lien avec une quelconque tradition. Quelle différence faire, alors, entre le simple bricolage, l'artisanat et la pratique d'un art profondément enraciné dans la culture populaire ?

Certes, cet ouvrage sera sans doute utile, comme le souligne Christian Denis dans sa préface, pour « les collectionneurs, amateurs, antiquaires [...] » qui en feront un guide de terrain. Le sera-t-il autant pour les muséologues et les chercheurs qui tentent d'aborder le phénomène en le débarrassant des parasites « de naïveté, d'archaïsme, de primitivisme » qui entachent le concept (Cuisenier, L'Art populaire en France, 1975)?

Bernard Genest

Société québécoise d'ethnologie

Mooney, Fraser. Jerome. Solving the Mystery of Nova Scotia's Silent Castaway. Halifax, Nimbus, 2008, 178 p. ISBN 978-1-55109-686-5.

La quatrième de couverture de cette plaquette annonce que ce récit est le résultat d'une longue recherche, qu'il présente des informations " inédites » démontrant «la vérité » sur l'identité de Jérôme, l'homme-mystère de la baie Sainte-Marie, abandonné sans jambes sur la plage de Sandy Cove en septembre 1863. Si cette affirmation est plus que contestable, l'ouvrage de Mooney (formé en journalisme et en histoire, apprend-on sur la jaquette arrière) est néanmoins divertissant et accessible à un large public.

Mooney a constitué un récit narratif enlevant et engageant. Il emploie un style haletant et un vocabulaire impressionniste, donnant vie aux personnages et créant une trame narrative emplie de suspens, qui accroche le lecteur à la manière d'un polar. La trame narrative est simple : la Nouvelle-Écosse est un lieu riche en mystères maritimes et en légendes, et Jérôme s'y imbrique parfaitement. Comme dans un bon polar, Mooney met en scène, d'un court chapitre à l'autre, le choc de la découverte de Jérôme à Sandy Cove, les efforts des résidents pour découvrir son identité, son déménagement à Météghan, puis à Saint-Alphonse, sa mise en spectacle, pour enfin présenter la redécouverte des informations démontrant que Jérôme et le " Frozen Man » de Chipman, Nouveau-Brunswick, étaient probablement la même personne. 
Il conclut enfin sur l'héritage de Jérôme après son décès en 1912.

Comme le récit de Mooney tient autant du roman que de l'essai, l'auteur mélange souvent la légende et les faits avérés. Les documents historiques sont avares de détails et Mooney met de la chair sur leur squelette en insérant les éléments de la légende sur Jérôme, de même qu'une part importante de rumeurs populaires, la plupart non avérées par les documents. Pour apporter des réponses à ses lecteurs, Mooney doit broder et mettre en scène les quelques faits certains, puis remplir les silences de la documentation avec de la spéculation et des anecdotes biographiques plus ou moins liées à Jérôme. Le tout est divertissant, mais souvent imprécis. L'auteur utilise indistinctement les documents d'époque et les articles de journaux de la fin du $\mathrm{Xx}^{\mathrm{e}}$ siècle et ne semble faire aucune analyse critique de ces derniers. De même, les citations intégrées dans le texte sonnent parfois comme des éléments de dialogue plutôt que des références documentaires, surtout lorsque l'auteur laisse entendre que ces citations sont le fruit d'entrevues. Les circonstances de ces rencontres n'étant jamais précisées, il est impossible de juger de leur exactitude. Mooney ne s'embarrasse pas de notes infrapaginales, préférant des références fragmentaires, à l'occasion. Si ceci allège considérablement le style du texte et plaira aux lecteurs non avertis, le silence sur la provenance des informations rend l'analyse du travail plus que difficile. En fait, seules les personnes intimement familières avec tout le corpus documentaire sur Jérôme pourraient évaluer cet ouvrage.

Ayant fait les mêmes recherches que Mooney et publié nos résultats sur la toile en 2007 (www.mysterescanadiens.ca), Lise A. Robichaud et moimême avons examiné tous les documents mentionnés ou cités par l'auteur dans son texte et dans la courte bibliographie à la fin de l'ouvrage. Il est donc possible d'affirmer que Mooney ne fait pas d'erreur factuelle, ni d'erreur majeure d'interprétation, même s'il donne beaucoup trop d'importance à la légende, surtout aux éléments canoniques de celle-ci. L'auteur donne énormément d'importance aux trois mots que Jérôme aurait prononcés au cours de ses quatre décennies en Acadie : Jérôme, Triestre et Colombo. Comme la légende prétend que ce dernier mot indiquait le vaisseau ayant transporté l'inconnu en terre nord-américaine, Mooney part à la recherche d'un navire portant ce nom. Il propose le schooner Columbus arrivé à Halifax à l'hiver 1859. Les détails reliés à ce bateau sont les seules informations proprement « inédites » de cet ouvrage (chapitre 11). Elles ne figurent pas dans notre site parce que nous les avions rejetées comme une fausse piste. Toutes les autres découvertes, incluant les liens entre le «Frozen Man» et Jérôme, apparaissent dans notre site publié un an avant son livre. Les autres conclusions de Mooney correspondent aux nôtres dans l'ensemble, même lorsqu'il emprunte parfois des stratégies différentes aux nôtres. Par exemple, 
dans un effort pour comprendre le silence de Jérôme et son amputation, nous avions contacté un médecin spécialiste, le docteur Ian A. Cameron, professeur à Dalhousie. Ce dernier a produit deux textes, sur les raisons probables des problèmes mentaux de notre homme et sur les techniques chirurgicales de l'époque, qui ont été annexés à notre site. Mooney, pour sa part, se contente de compiler des informations sur une explication plausible des problèmes communicatifs de Jérôme, qu'il tire d'ouvrages de médecine populaire (p. 146-152).

C'est ici que le bât blesse : comme notre travail vise ouvertement à favoriser la création de travaux comme Jerome : Solving the Mystery of Nova Scotia's Silent Castaway, il est surprenant que Mooney n'ait pas utilisé la masse documentaire que nous avons mise en ligne à la disposition de tous. Le site n'apparaît pas en bibliographie. L'auteur ne réfère à nos conclusions qu'une seule fois, en rapport avec le prénom probable de Jérôme (p. 120). Enfin, lorsqu'il mentionne notre travail directement, dans le dernier chapitre, il consacre plus d'espace aux fêtes entourant le lancement du site qu'à son contenu (p. 168). C'est d'autant plus dommage que nous avons inclus de nombreuses traductions anglaises de rares textes en langue française et de témoignages en dialecte acadien, alors que l'auteur se contente strictement de sources anglophones.

En somme, Jerome : Solving the Mystery of Nova Scotia's Silent Castaway démontre toutes les forces et les faiblesses typiques à l'histoire populaire et journalistique. Elle est agréable à lire et entraîne le lecteur dans une aventure historique. Sans aucun doute, cet ouvrage fera effet. Cependant, Mooney propose des certitudes à son public, même lorsque la documentation ne le permet pas. La prudence requise à la discipline historique y est absente. Comme les certitudes sont plus attrayantes que les silences, le récit de Mooney prendra sans doute dans l'avenir une grande place dans la longue lignée légendaire sur Jérôme.

Caroline-Isabelle Caron

Queen's University

Oriol, Carme et Josep-Maria Pujol. Index of catalan folktales. Helsinki, Academia Scientiarum Fennica, «Folklore Fellows Communications 》 $\mathrm{n}^{\circ} 294,2008,313$ p. ISBN 978-951-41-1015-3.

Carme Oriol et Josep-Maria Pujol, professeurs au département d'Études catalanes de 1'Université Rovira e Virgil (Tarragona, Catalunya) nous présentent ici le fruit d'un travail entamé depuis plus de trente ans : un index 